\title{
Superlensing Microscope Objective Lens
}

\author{
Bing Yan, ${ }^{1}$ Zengbo Wang, ${ }^{1,}{ }^{*}$ Alan L. PARker, ${ }^{2}$ Yu-Kun Lal, ${ }^{3}$ P. John \\ THOMAS, ${ }^{4}$ LIYANG YUE, ${ }^{1}$ AND JAMES N MONKS ${ }^{1}$
}

\author{
${ }^{1}$ School of Electronic Engineering, Bangor University, LL57 1UT, Bangor, UK \\ ${ }^{2}$ Division of Cancer and Genetics, Cardiff University School of Medicine, CF14 4XN, Cardiff, UK \\ ${ }^{3}$ School of Computer Science and Informatics, Cardiff University, CF24 3AA, Cardiff, UK \\ ${ }^{4}$ School of Chemistry, Bangor University, LL57 2UW, Bangor, UK \\ *Corresponding author: z.wang@bangor.ac.uk
}

Received XX Month XXXX; revised XX Month, XXXX; accepted XX Month XXXX; posted XX Month XXXX (Doc. ID XXXXX); published XX Month XXXX

\begin{abstract}
Conventional microscope objective lenses are diffraction limited; they cannot resolve subdiffraction features of size smaller than 250-300 $\mathrm{nm}$ under white lighting condition. New innovations are required to overcome such limit. In this paper, we propose and demonstrate a new superlensing objective lens which possesses a resolution of $100 \mathrm{~nm}$, offering two times resolution improvement over conventional objectives. This is accomplished by integrating a conventional microscope objective lens with a superlensing microsphere lens using a customized lens adaptor. The new objective lens was successfully demonstrated for label-free super-resolution imaging of $100 \mathrm{~nm}$ features in engineering and biological samples, including a Blu-ray disc sample and adenoviruses. Our work opens a new door to develop a generic optical superlens, which may transform the field of optical microscopy and imaging.
\end{abstract}

OCIS codes: (100.6640) Superresolution, (180.4243) Near-field microscopy, (180.5810) Scanning microscopy, (170.3880) Medical and biological imaging.

\section{INTRODUCTION}

Objective lens is the core element of an optical microscope, whose resolution is limited by the classical diffraction law as first formulated by the German physicist Ernst Abbe in 1873: The minimum distance, $d$, between two structural elements to be imaged as two objects instead of one is given by $d=\lambda /(2 \mathrm{NA})$, where $\lambda$ is the wavelength of light and NA is the numerical aperture of the objective lens [1]. The physical root stems from the loss of exponentially-decaying evanescent waves in the far field that carry high spatial frequency subwavelength information of an object [2].

The development of Near-field Scanning Optical Microscope (NSOM) opens the door to super-resolution research. It uses a tiny tip positioned close to an object's surface to collect evanescent waves in near-field [3]. Scanning the sample generates a super-resolution image whose resolution is determined by tip size instead of wavelength. Since the late 1990s, owing to the arising of metamaterials, nanophotonics, and plasmonics, the field of super-resolution research grows extremely rapidly. A variety of super-resolution techniques were developed, including for example Pendry-Veselago superlens [2, 4], optical superoscillatory lens [5], time-reversal imaging [6], Maxwell fisheye [7], scattering lens [8], and most famously, the super-resolution fluorescence microscopy techniques [9-11] which won the 2014 Nobel Prize. However, none of these techniques would operate under white lighting sources, e.g. the conventional Halogen lamps or recent white LEDs, whose radiation covers a broadband electromagnetic spectrum. Monochromatic lasers are required. The solution to white light superresolution appears in 2011, when we first reported that microsphere can work as a superlens under white lights and achieve a resolution between 50-100 nm [12-14]. The super-resolution arises from microsphere's ability to focus light beyond diffraction limit, a phenomenon known as 'photonic nanojet' $[15,16]$, and near-field collection of evanescent waves by the microspheres in contacting with imaged objects. This technique is label-free and has been advanced since by a number of groups across the world, including for example the developments of confocal microsphere nanoscopy [14, 17], solid immersion microsphere nanoscopy $[18,19]$, microfiber nanoscopy [20], subsurface nano-imaging [21, 22], and new designs of microsphere superlens [23, 24]. Very recently, we demonstrated new superlens made from high-index nanobeads [25] and create the world's first biological superlens using spider silks [26]. For better understanding of the field, please refer to our recent review article [14].

The imaging window of a microsphere lens is often very small, typically a few micrometers only. This requires a scanning operation of the microsphere superlens to generate a complete image of a sample. There are a few demonstrations in the literature. Krivitsk et al. attached a fine glass micropipette to the microsphere lens to scan the particle [27]. Li et al. designed a 'swimming lens' technique in which the microsphere lens was propelled and scanned across the sample 
surface by chemical reaction force in a liquid [28]. We proposed a coverslip superlens by encapsulating high-index microspheres $\mathrm{BaTiO}_{3}$ or $\mathrm{TiO}_{2}$ ) inside a transparent host material (such as PMMA and PDMS)[29]. The microsphere was completely immersed inside the encapsulation material with its bottom touching the coverslip surface (see Fig. 1a side view). This concept was also explored extensively by Darafsheh and Astratov et al. in the US [18, 30, 31]. The coverslip superlens can be manually manipulated in a way similar to classical coverslip, offering the freedom to position particle lens at desired location. Scanning of microsphere superlens, however, requires synchronization with microscope objective for full super-resolution image construction. This is difficult to achieve with existing design of coverslip superlens which is separated from objective lens. In this paper, we propose an improved design which solves the synchronization problem between coverslip superlens and objective lens. The idea is simple yet effective, using a custom-made lens adaptor to integrate these two lenses to form a superlensing microscope objective lens.

\section{SUPERLENSING MICROSCOPE OBJECTIVE}

(a)
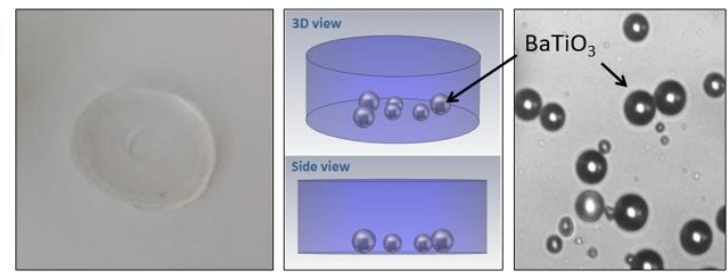

(b)

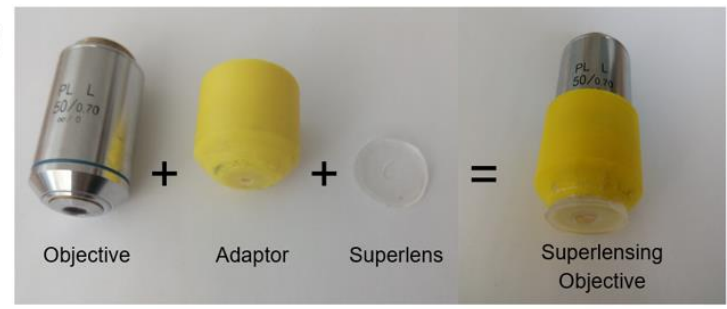

(c)

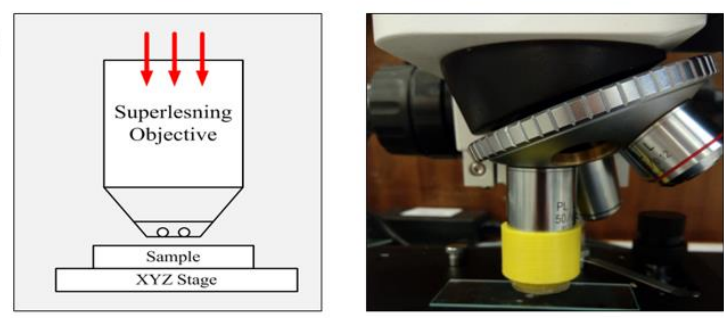

Fig. 1. Superlensing objective lens. (a) The $\mathrm{BaTiO}_{3}$ superlens was fabricated by encapsulating a monolayer of $\mathrm{BaTiO}_{3}$ microsphere (3-80 $\mu \mathrm{m}$ diameter) inside a PDMS material. (b) the super objective was made by integrating a conventional microscope objective lens (e.g. 50x, NA: 0.70 , or $100 x, \mathrm{NA}: 0.95$ ) with a $\mathrm{BaTiO}_{3}$ microsphere superlens using a 3D printed adaptor (c) experimental configuration for superresolution imaging using developed objective which was fitted onto a standard white light optical microscope.

The key concept and design of superlensing microscope objective is illustrated in Fig. 1(b) and (c). A conventional microscope objective (OB) lens with a magnification factor between $40 \mathrm{x}$ and 100x, NA between 0.7 and 0.95 was selected. A lens adaptor was designed in CAD software (e.g. Solidworks) and then printed with a 3D plastic printer (model: Prusia I5). The adaptor has a tube size fit to the objective lens tube, with reasonable friction allowing up-down adjustment. A coverslip superlens (Fig.1a) was bonded to the bottom end of the adaptor using high-adhesive glue. This results in an integrated objective lens consisting of conventional OB and a Coverslip Microsphere Superlens (CMS). The imaging resolution will be determined by the coverslip superlens while the conventional objective lens provides necessary condition for illumination. The obtained superlensing lens can be easily fitted to any existing conventional microscopes; in this study, we used two brands of optical microscopes to prove the lens's flexibility (Olympus BX60 and a lowcost ICM100 microscope) in usage.

The scanning was performed using a high-resolution nano-stage (model: PI P-611.3 Nanocube), with $1 \mathrm{~nm}$ resolution in XYZ direction, travel range $100 \mu \mathrm{m}$. Samples (Blu-ray disc, semiconductor chip, virus on glass slider) were firmly bonded to the nano-stage using highstrength double-sided sticking tape. In experiments, the superlensing objective lens was kept static and the underlying nano-stage moved and scanned the samples across the objective lens. The imaging process was video recorded using a high-resolution camera (ToupCAM UCMOS 14000KPA). The video was then analysed and frames were used to generate a stitched image of the sample. The focusing properties of developed superlens were analysed using classical Mie theory.

\section{EXPERIMENTS}

\section{A. Static Imaging}

The superlensing objective lens was carefully adjusted to maximum imaging contrast and image quality. The main adjustments include the followings: (1) the lens adaptor was adjusted so that the top objective lens will pick up virtual image generated by the bottom coverslip microsphere superlens. (2) Light illumination angle was adjusted, roughly an angle between 10-40 degrees will produce enhancement in image quality, because the magnification factor is increasing with incident angles and there is a compromise between image quality and magnification factor [26]. Best imaging resolution is obtained in contacting mode where lens and samples contact with each other. The resolution was found decreasing rapidly when lens moved away from the sample surface, at about the wavelength distance $(\sim 600 \mathrm{~nm})$ away the super-resolution was completely lost (due to loss of evanescent wave contribution) which indicates the near-field nature of the technique. This inversely poses a technical challenge in scanning imaging as will be discussed below.

In the contacting mode, we carried out super-resolution imaging of several different samples, including Blu-ray disc with $100 \mathrm{~nm} / 200 \mathrm{~nm}$ features and adenoviruses (replication deficient human adenovirus serotype 5 vector) with size about 90-100 nm. Noting superlensing objective lens in these tests could be slightly different from each other, with an aim of demonstrating the ability to achieve super-resolution with different lens configurations. As previously mentioned, the overall resolution is determined by Coverslip Microsphere Superlens (CMS) which consists of $\mathrm{BaTiO}_{3}$ microsphere $(3-80 \mu \mathrm{m})$ inside PDMS sheet. Figures 2(a-c) demonstrate Blu-ray disc imaging using the developed superlensing objectives. In Fig. 2(a), the SEM image reveals that the disk consists of $200 \mathrm{~nm}$ gratings separated $100 \mathrm{~nm}$ away. Figs. 2(b-c) show the images obtained using Olympus BX60 microscope equipped with developed superlensing objective lenses with the following parameters: Fig. 2(b) with $\mathrm{OB}(100 \mathrm{x}, \mathrm{NA0.90})+\mathrm{CMS}\left(67 \mu \mathrm{mBTiO}_{3}\right.$ in PDMS) and Fig. 2(c) with OB (100x, NA0.90) + CMS $\left(22 \mu \mathrm{m} \mathrm{BaTiO}_{3}\right.$ in PDMS). As can be seen, both lenses can clearly resolve the $100 \mathrm{~nm}$ features, which is beyond the classical diffraction limit of $300 \mathrm{~nm}$. The smaller diameter $\mathrm{BaTiO}_{3}$ superlens in Fig. 2(c) offers a larger magnification factor $(\mathrm{M} \sim 6 \mathrm{x})$ over the larger sized $\mathrm{BaTiO}_{3}$ superlens 
(M 2x) in Fig. 2(b). This is caused by the shorter focal length of a smaller sized microsphere lens in virtual imaging mode [12]. The central zone of the resulting image appears a bit over-exposed in Fig. 2(b) due to reflected beams by the Blu-ray substrate. This phenomenon is less obvious in smaller particles (Fig. 2c). In microsphere superlens imaging, artificial images are always an issue and one should pay particular attention to it. The artificial images can be excluded by rotating the imaging samples.
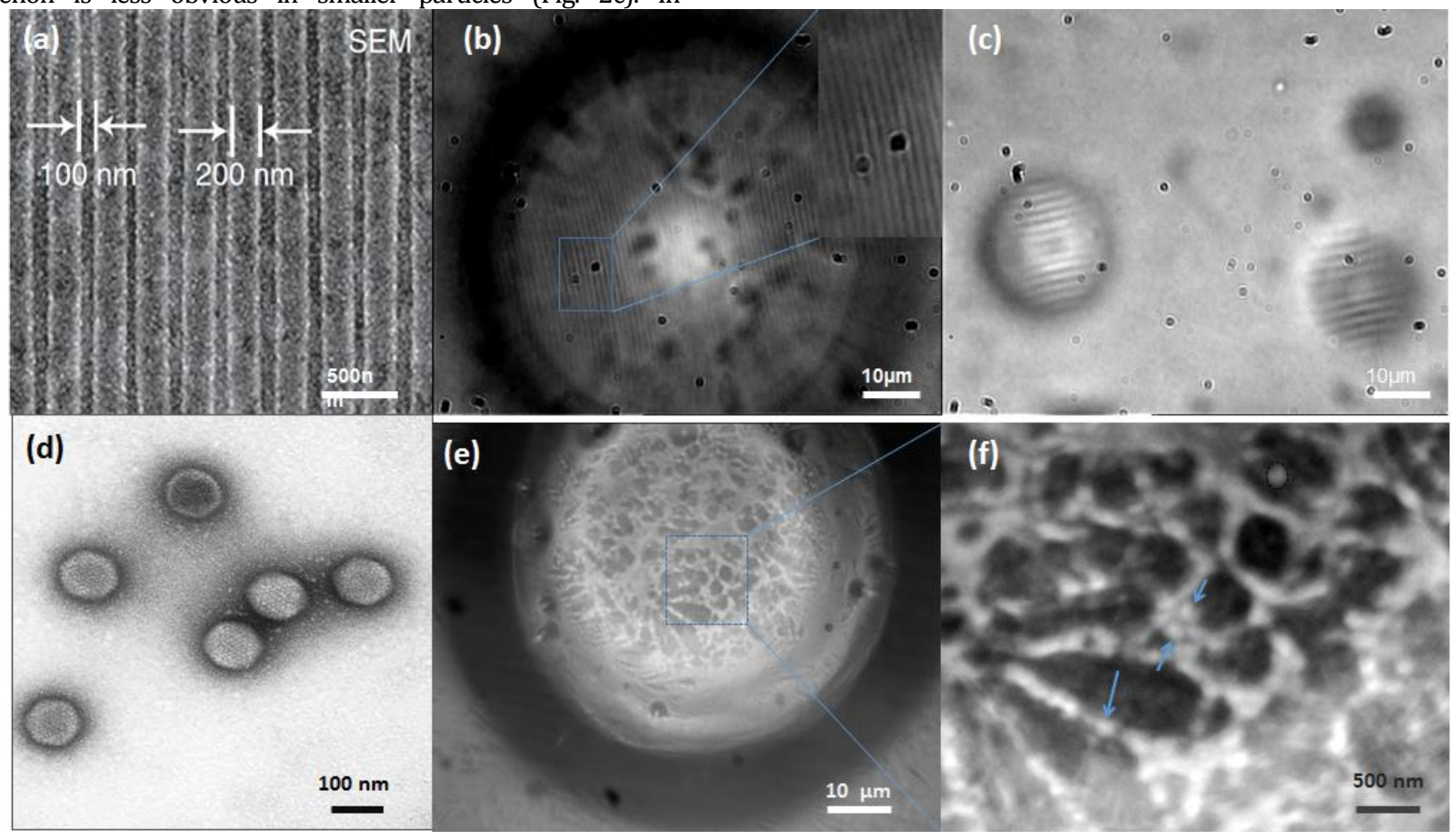

Fig. 2. Static super-resolution imaging of Blu-ray and virus by superlensing objective lens. (a) SEM image of Blu-ray disc. (b) $67 \mu \mathrm{m}$ BaTiO3 superlens imaging. (c) $18 \mu \mathrm{m}$ BaTiO3 superlens imaging. (d) SEM of adenovirus. (e) $70 \mu \mathrm{m}$ BaTiO3 superlens imaging of virus cluster. (f) Enlarged view of section of $(\mathrm{e})$.

Let's turn our eye on another sample, the adenoviruses sample. These virus particles are nearly spherical in shape with diameter around $90-100 \mathrm{~nm}$ (Fig. 2d), and they are sub-diffraction-limited and is difficult to be observed using conventional microscopes. Figure 2(e) shows a super-resolution image obtained using a superlensing objective lens formed by $80 \mathrm{x}$, NA0.90 objective and $70 \mu \mathrm{m} \mathrm{BaTiO}$ microsphere. The viruses are aggregated in clusters in our sample, but it was possible to isolate them by zooming in the image. The magnified particle size is about $200 \mathrm{~nm}$ in Fig. 2(f), which means the real virus size is around $100 \mathrm{~nm}$ since the $70-\mu \mathrm{m} \mathrm{BaTiO}_{3}$ superlens offers a magnification factor around 2 under the experimental condition. Compared to Fig. 2(b), here the central zone doesn't have a bright spot due to reflection by underlying substrate, this is because virus particles are deposited on a glass slider which is transparent and has much less reflection compared to a Blu-ray disc.

\section{B. Scanning Imaging and Image Stitching}

In our superlensing objective lens, the microsphere superlens is synchronized with objective lens. During the scanning operation, both parts of the lens will move together simultaneously, and their relative position is kept constant. This is beneficial to scanning imaging since it ensures that the same particle lens is used in imaging. It is also possible to have multiple particle lenses in parallel for simultaneous scanning imaging. In experiments, we evaluated scanning imaging of different samples, including the adenovirus samples. However, due to lenssample contacting requirements, we had some difficulty in scanning imaging of virus samples, since the virus particles were dragged by the lens during scanning. This is due to the virus particles not being well fixed onto the glass slides. This may not be the case for cell samples which could be immobilized on glass slides and is the plan of our next step experiments. In this study, scanning imaging was performed with well-structured semiconductor IC chip and Blu-ray disk sample to demonstrate the feasibility of scanning imaging using developed superlensing objective lens. This was shown in Fig. 3. To reduce the friction between the lens and the sample, we evaluated different lubricant medium including DI water, IPA, silicone oil, and WD40. WD40 produces the best lubrication effect so it was used in Fig. 3 scanning experiments. The IC chip in Fig. 3(b) has features of $200 \mathrm{~nm}$ and $400 \mathrm{~nm}$, which are easy to be observed using the new lens. The scanning process was video recorded (Supplementary video 1). Comparing Fig. 3(c), (d), (e), we can see the scanning takes place along the indicated scanning direction in Fig. 3(c), and high-resolution images were taken at different spatial positions. Stitching images produces a larger-sized picture covering scanned sample regions of interest following the scanning path (see demonstration in insets of Figs. 3d-e). The in-house developed image stitching code based on MATLAB language works automatically for a sequence of frames with moderate movement between adjacent frames. The location and size of a superlens imaging region in the first image frame are specified. In the subsequent frames, the location can change gradually. To compensate for this, with the assumption that superlens imaging regions are in focus and thus contain more image features, the algorithm searches in the neighborhood of the location from the previous frame to find the region with most significant total edge strengths. For each extracted region, radial distortion correction is applied. The correction parameter is estimated based on images with 
regular grid structure (e.g. an image on the IC chip). From the first extracted region, the images are stitched sequentially, and the optimal offset between adjacent extracted regions is calculated based on maximizing the normalized cross correlation. This criterion works more effectively than e.g. sum of squared differences because it copes with variation of image brightness better. The stitched images are obtained by integrating pixels from all the extracted regions with

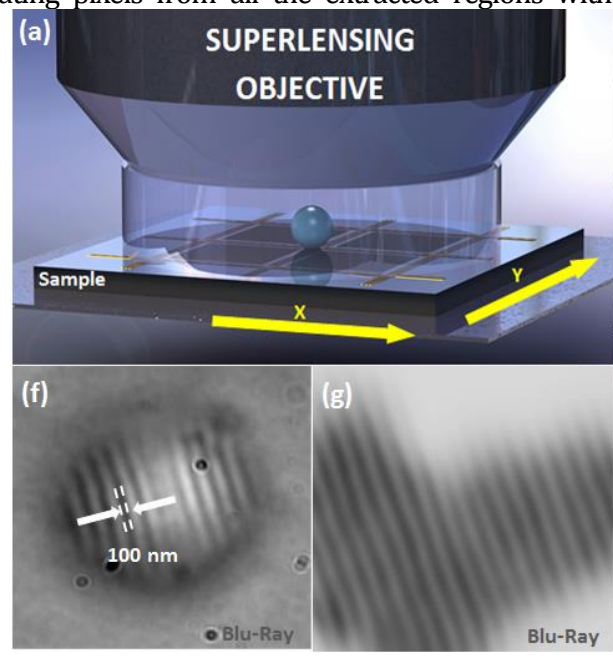

corresponding offsets applied. To avoid visible boundaries between regions from different frames, alpha blending is used such that for the stitched image pixels where multiple source regions overlap, a combination of their contributions is used. Similar scanning experiments were performed with Blu-ray disk sample as well (Fig. 3f), where stitched image was shown in Fig. 4(g).

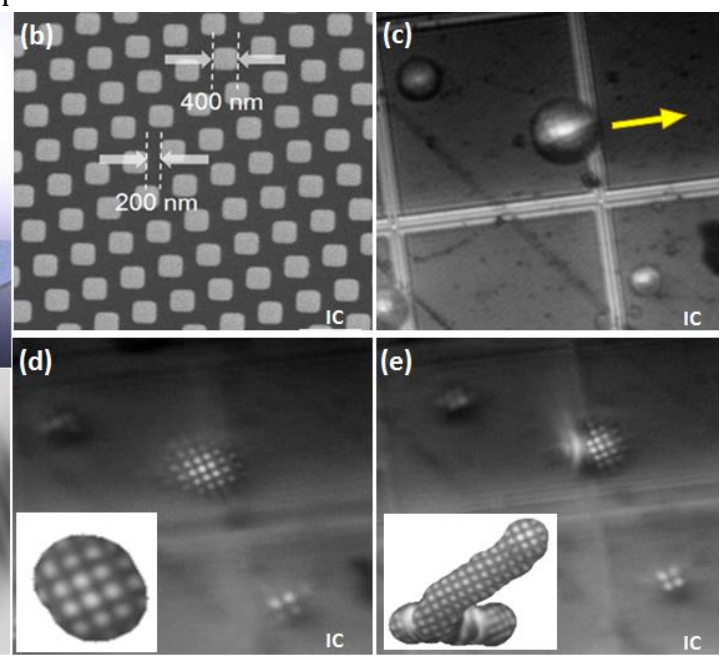

Fig. 3. Scanning super-resolution imaging and image stitch. (a) Scanning schematics. (b) Scanning direction of IC chip sample (c) SEM of IC chip. (d) IC scanning image position 1. (e) IC scanning position 2. The insets show stitched image following scanning paths. (f) Blu-ray sample imaged by microsphere. (g) stitched image of Blu-ray scanning.

\section{MECHANISM AND DISCUSSION}

Light interaction with spherical particles can be simulated using the classical Mie theory. Figure 4 shows examples of calculated electric field intensity $|\mathrm{E}|^{2}$ distribution across cross-sectional plane of an $18 \mu \mathrm{m}$ and a $67 \mu \mathrm{m} \mathrm{BaTiO}_{3}$ microsphere $(\mathrm{n}=1.90)$ embedded in PDMS $(\mathrm{n}=1.40)$ under different illumination wavelengths, covering $400 \mathrm{~nm}$ to 700 spectrum range of a typical white lighting source. The corresponding size parameter for each case, defined as $\mathrm{q}=\pi \mathrm{D} / \lambda$, where $\mathrm{D}$ is the particle diameter and $\lambda$ the wavelength, was also indicated in the figure. A video was provided in the Supplementary (video 2) offering a systematic view of the evolution of field distribution for size parameters $0.1 \leq q \leq 628$, with step accuracy of $\Delta q=0.1$. From Fig. 4 we can see, besides the well-known photonic nanojet focusing modes by microspheres (Figs.4a, 4c, 4d,4f), strong internal focusing modes featuring hugely enhanced intensity peaks inside the particle could be excited at particular wavelengths (Figs. 4b, 4e). All these modes will play a role in super-resolution image formation.

The imaging sample in contact with microsphere is illuminated by the microsphere lens; super-resolution arises from the strong nearfield interaction between the microsphere lens and underlying nanoobjects, which leads to the conversion of surface-bounded high spatial frequency evanescent waves into propagating waves [14, 32]. Such interaction process is quite complex and is out of the scope of Mie theory. An example calculation for the coupled microsphere-substrate system was demonstrated in a previous publication [14] using a fullwave simulation approach. It was noted that such conversion process is sensitive to the substrate properties and the gap distance between lens and object. The technique requires gap distance below wavelength scale and ideally a zero distance gap is desired [14]. Besides substrate effect, other factors such as different-NA illumination, incident illumination angle, and microscope image plane have been found also influencing the experimental resolution and magnification. These uncertainties are challenges, but also opportunities for next-generation dielectric superlens development. For example, inspired by enhancing the lens and sample evanescent coupling for a better super-resolution, we recently used high-index $\mathrm{TiO}_{2}$ nanoparticle as building block to form a mouldable micro-sized lens which was deposited on imaging sample, leading to perfect contacting with imaging sample and meanwhile supporting extremely high evanescent-to-propagation conversion efficiency. Such new design of superlens has shown greatly improved imaging contrast and field of view over all previously reported microsphere superlens, and has a record white light super-resolution of $45 \mathrm{~nm}$ [25].

Our work reported here laid down a solid foundation for further development of superlensing objective lens technology and it is the first kind of such lens in the literature. We are expecting the key problem of contacting scanning will be circumvented in future design, and a contactless superlensing objective lens will be developed. This may be achieved by combining optical superoscillatory mask design with existing superlensing objective design. 

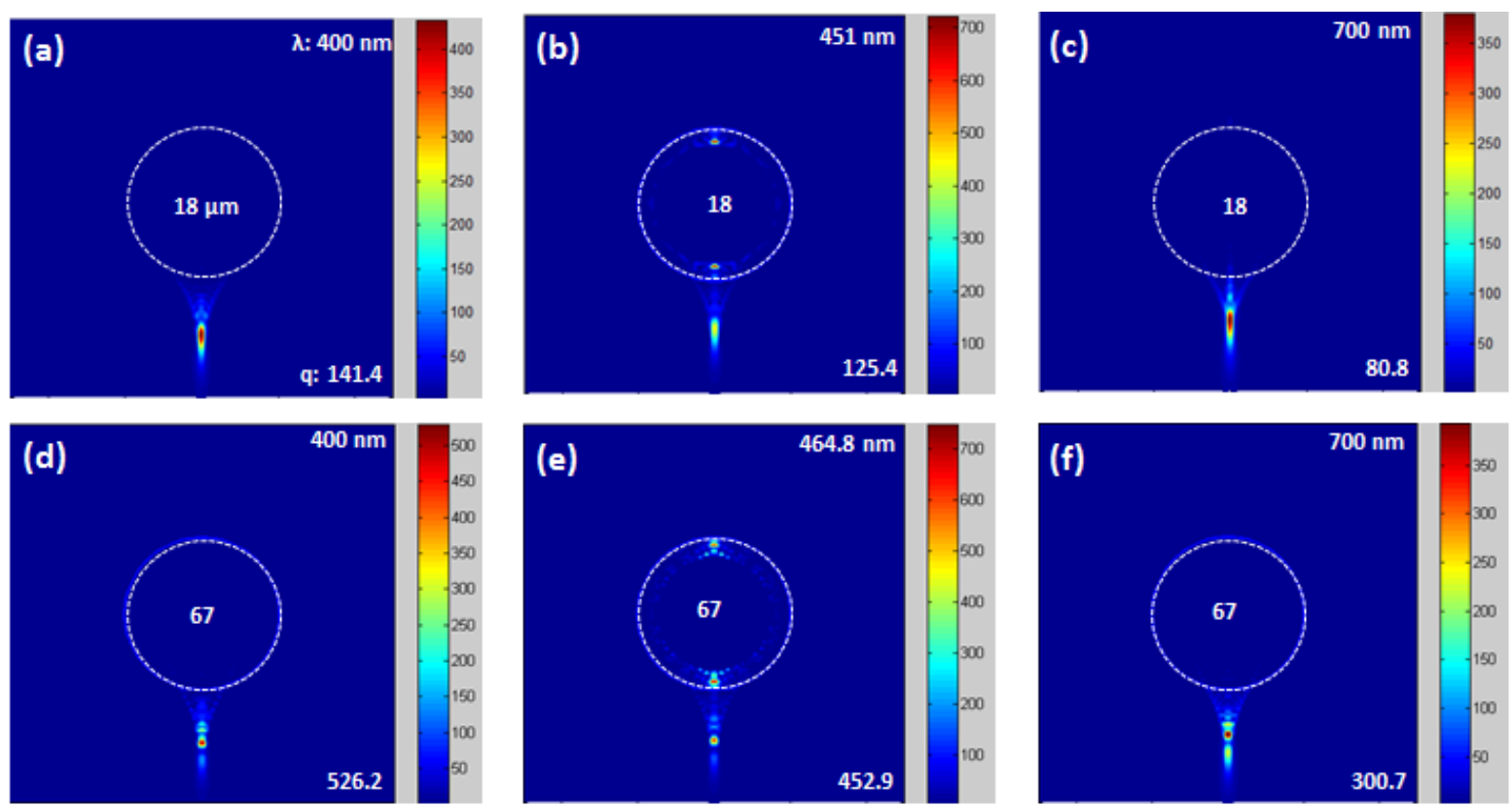

Fig. 4. Electric field intensity $\left(|\mathrm{E}|^{2}\right)$ distribution in the cross sectional plane of $18 \mu \mathrm{m}$ and $67 \mu \mathrm{m}$ diameter BaTiO${ }_{3}$ microsphere embedded in PDMS material illuminated by different visible wavelengths. The light is incident from top to bottom, with electric field polarized from left to right. $\mathrm{q}$ is the size parameter defined as $\mathrm{q}=\pi \mathrm{D} / \lambda$, where $\mathrm{D}$ is the particle diameter and $\lambda$ the wavelength.

\section{CONCLUSION}

To summarise, we have demonstrated a new type of microscope objective lens, a superlensing objective lens that integrates a conventional objective lens and a coverslip-like microsphere superlens. The new lens has advantages in usability and is able to image the sample with super-resolution in both static and scanning modes. A resolution of $100 \mathrm{~nm}$ under white light illumination has been demonstrated. The developed superlensing objective lens has potential to be further developed into a commercial product which would transform an existing microscope into a nanoscope.

Acknowledgment. The authors thank funding supports from Welsh Crucible Grant (Bangor University: 006311 Cardiff University: 510435) and Sêr Cymru National Research Networking Advanced Engineering and Materials, UK (NRN113, NRNF66).

\section{References}

1.

E. Abbe, "Beitrage zur Theorie des Mikroskops und der mikroskopischen Wahrnehmung," Archiv Microskop. Anat. 9, 413 (1873).

2. $\quad X$. Zhang and Z. W. Liu, "Superlenses to overcome the diffraction limit," Nature Materials 7, 435-441 (2008).

$3 . \quad$ D. W. Pohl, W. Denk, and W. Lanz, "Optical stethoscopy: image recording with resolution ladmda/20," Appl. Phys. Lett. 44, 651 (1984).

$4 . \quad$ J. B. Pendry, "Negative Refraction Makes a Perfect Lens," Phys. Rev. Lett. 85, 3966 (2000).

5. E. T. Rogers, J. Lindberg, T. Roy, S. Savo, J. E. Chad, M. R. Dennis, and N. I. Zheludev, "A super-oscillatory lens optical microscope for subwavelength imaging," Nat. Mater. 11, 432-435 (2012).

6. M. Davy, J. G. Minonzio, J. de Rosny, C. Prada, and M. Fink, "Influence of Noise on Subwavelength Imaging of Two Close Scatterers Using Time Reversal Method: Theory and Experiments," Prog. Electromagn. Res. 98, 333-358 (2009).
7.

8.

9.

.

V. Smolyaninova, C. Jensen, W. Zimmerman, A. Johnson, D. Schaefer, and I. Smolyaninov, "Lithographically Fabricated Magnifying Maxwell Fisheye Lenses," Photonics 3(2016).

J. H. Park, C. Park, Y. H. Cho, and Y. Park, "Scattering super-lens: subwavelength light focusing and imaging via wavefront shaping in complex media," 2014 Conference on Lasers and Electro-Optics (Cleo) (2014). stimulated emission: stimulated-emission-depletion fluorescence microscopy," Opt. Lett. 19, 780-782 (1994).

E. Betzig, G. H. Patterson, R. Sougrat, O. W. Lindwasser, S. Olenych, J. S. Bonifacino, M. W. Davidson, J. Lippincott-Schwartz, and H. F. Hess, "Imaging intracellular fluorescent proteins at nanometer resolution," Science 313, 1642-1645 (2006).

S. T. Hess, T. P. K. Girirajan, and M. D. Mason, "Ultra-high resolution imaging by fluorescence photoactivation localization microscopy," Biophys. J. 91, 4258-4272 (2006).

Z. Wang, W. Guo, L. Li, B. Luk'yanchuk, A. Khan, Z. Liu, Z. Chen, and M. Hong, "Optical virtual imaging at $50 \mathrm{~nm}$ lateral resolution with a whitelight nanoscope," Nat. Commun. 2, 218 (2011).

Z. B. Wang and L. Li, "White-light microscopy could exceed $50 \mathrm{~nm}$ resolution," Laser Focus World 47, 61-64 (2011).

Z. B. Wang, "Microsphere super-resolution imaging," in Nanoscience, J. T. Paual O Brien, ed. (Royal Society of Chemistry, 2016), pp. 193-210.

Z. G. Chen, A. Taflove, and V. Backman, "Photonic nanojet enhancement of backscattering of light by nanoparticles: a potential novel visible-light ultramicroscopy technique," Opt. Express 12, 1214-1220 (2004).

Y. F. Lu, L. Zhang, W. D. Song, Y. W. Zheng, and B. S. Luk'yanchuk, "Laser writing of a subwavelength structure on silicon (100) surfaces with particle-enhanced optical irradiation," JETP Lett. 72, 457-459 (2000).

Y. Yan, L. Li, C. Feng, W.Guo, S.Lee, and M.H.Hong, "MicrosphereCoupled Scanning Laser Confocal Nanoscope for Sub-Diffraction-Limited Imaging at $25 \mathrm{~nm}$ Lateral Resolution in the Visible Spectrum," Acs Nano 8, 1809-1816 (2014). Astratov, "Advantages of microsphere-assisted super-resolution imaging technique over solid immersion lens and confocal microscopies," Appl. Phys. Lett. 104, 061117 (2014). 
19. A. Darafsheh, G. F. Walsh, L. Dal Negro, and V. N. Astratov, "Optical super-resolution by high-index liquid-immersed microspheres," Appl. Phys. Lett. 101, 141128 (2012)

20. A. Darafsheh, G. Wu, S. Yang, and J. C. Finlay, "Super-resolution optical microscopy by using dielectric microwires," in Three-Dimensional and Multidimensional Microscopy: Image Acquisition and Processing XXIII, (SPIE, 2016), $97130 \mathrm{U}$

21. G. Q. Gu, R. Zhou, H. Y. Xu, G. X. Cai, and Z. P. Cai, "Subsurface nanoimaging with self-assembled spherical cap optical nanoscopy," Opt. Express 24, 4937-4948 (2016).

22. R. Ye, Y. H. Ye, H. F. Ma, J. Ma, B. Wang, J. Yao, S. Liu, L. L. Cao, H. H. Xu, and J. Y. Zhang, "Experimental far-field imaging properties of a similar to 5-mu m diameter spherical lens," Opt. Lett. 38, 1829-1831 (2013).

23. B. Yan, L. Y. Yue, and Z. B. Wang, "Engineering near-field focusing of a microsphere lens with pupil masks," Opt. Commun. 370, 140-144 (2016).

24. L. Y. Yue, B. Yan, and Z. B. Wang, "Photonic nanojet of cylindrical metalens assembled by hexagonally arranged nanofibers for breaking the diffraction limit," Opt. Lett. 41, 1336-1339 (2016).

25. W. Fan, B. Yan, Z. B. Wang, and L. M. Wu, "Three-dimensional alldielectric metamaterial solid immersion lens for subwavelength imaging at visible frequencies," Sci. Adv. 2(2016).

26. J. N. Monks, B. Yan, N. Hawkins, F. Vollrath, and Z. B. Wang, "Spider Silk: Mother Nature's Bio-Superlens," Nano Lett. 16, 5842-5845 (2016).

$27 . \quad$ L. A. Krivitsky, J. J. Wang, Z. B. Wang, and B. Luk'yanchuk, "Locomotion of microspheres for super-resolution imaging," Sci Rep-Uk 3, 3501 (2013).

$28 . \quad$ J. X. Li, W. J. Liu, T. L. Li, I. Rozen, J. Zhao, B. Bahari, B. Kante, and J. Wang, "Swimming Microrobot Optical Nanoscopy," Nano Lett. 16, 6604-6609 (2016).

29. Z. Wang, "Improvements in and Relating to Lenses," PCT/GB2014/052578 (priority date: 2013-AUG-23) (2014).

30. K. W. Allen, N. Farahi, Y. Li, N. I. Limberopoulos, D. E. Walker, A. M. Urbas, V. Liberman, and V. N. Astratov, "Super-resolution microscopy by movable thin-films with embedded microspheres: Resolution analysis," Ann. Phys. 527, 513-522 (2015).

31. V. N. Astratov and A. Darafsheh, "Methods and systems for superresolution optical imaging usinghigh-index of refraction microspheres and micro-cylinders,," US patent application 2014/0355108 A1 published on December 4, 2014 (2014).

32. X. Hao, C. Kuang, Z. Gu, Y. Wang, S. Li, Y. Ku, Y. Li, J. Ge, and X. Liu, "From microscopy to nanoscopy via visible light," Light Sci Appl. 2, e108 (2013). 